\title{
Fault Diagnosis of A High Voltage TRANSMISSION LINE USING WAVEFORM MATCHING APPROACH
}

\author{
Ripunjoy Phukan ${ }^{1}$, Rishab K Gupta ${ }^{2}$, Sandeep Dadga ${ }^{3}$ and Ananthanaryan \\ Rathinam $^{4}$ \\ ${ }^{1,3,4}$ EEE Dept., SRM University, Kattankulathur, Chennai \\ ${ }^{2}$ ECE Dept., UT Dallas, Texas, USA
}

\begin{abstract}
This paper is based on the problem of accurate fault diagnosis by incorporating a waveform matching technique. Fault isolation and detection of a double circuit high voltage power transmission line is of immense importance from point of view of Energy Management services. Power System Fault types namely single line to ground faults, line to line faults, double line to ground faults etc. are responsible for transients in current and voltage waveforms in Power Systems. Waveform matching deals with the approximate superimposition of such waveforms in discretized versions obtained from recording devices and Software respectively. The analogy derived from these waveforms is obtained as an error function of voltage and current, from the considered metering devices. This assists in modelling the fault identification as an optimization problem of minimizing the error between these sets of waveforms. In other words, it utilizes the benefit of software discrepancies between these two waveforms. Analysis has been done using the Bare Bones Particle Swarm Optimizer on an IEEE 2 bus, 6 bus and 14 bus system. The performance of the algorithm has been compared with an analogous meta-heuristic algorithm called BAT optimization on a 2 bus level. The primary focus of this paper is to demonstrate the efficiency of such methods and state the common peculiarities in measurements, and the possible remedies for such distortions.
\end{abstract}

\section{KEYWORDS}

ATP, BBPSO, BAT, COMTRADE, Waveform Matching

\section{INTRODUCTION}

Distribution Systems use several intelligent electronic devices (IEDs) such as digital protection devices, modern metering devices and Remote Terminal Units. These devices are generally equipped with super-processors, which are capable of advanced computations within a shorter time scale. With such advanced metrics and computation, faster and improved fault detection and identification techniques can be investigated, for a possible implementation in real time scenario. Several methods in the past detect a fault in the distribution system through conventional approaches. For instance, a temporary interruption in power supply is dictated in terms of a power system fault. Several, Maintenance personals are deployed all over the affected area, for identification. Meanwhile, for an underground cable system, such facilities are rendered useless and so switching operations were widely practiced to identify the faulted section. Thus, the locating process is time consuming and the system becomes bulky. Due to these complications, a number of automated fault location methods have been introduced for the process of fault location. The fault methods for distribution networks are categorized as Impedance based 
International Journal on Soft Computing (IJSC) Vol.4, No.4, November 2013

methods and Other Fundamental Frequency Methods, High Frequency Components and Travelling Wave Based Methods, Knowledge-Based Methods, Artificial Neural Networks, Matching Approach, Hybrid methods, Wavelet transform and Magnetic field sensing coils. Quick fault detection can help protect equipment through faster disconnection of faulted lines before any significant cascaded damage is done. The reason behind a strategy for accurate fault location is to assist in removing potential sites for persistent faults and locate areas where faults could regularly occur, thus reducing the frequency and length of power outages. Hence, while many fault diagnosis schemes have been developed in the past, a variety of algorithms continue to be developed solely to perform this task more accurately and more effectively. Most faults in an Electrical system occur within a network of overhead lines as they are highly susceptible to vagaries of nature. More than $70 \%$ of the fault types belong to the genre of single-phase to ground faults caused due to lightning induced transient high voltage or from falling trees. In the overhead lines, tree contact caused by wind is a major cause for such faults along with double line to ground faults.

Several papers have reported surveys on evolutionary algorithms (EAs) and their applications in power systems [1]. Nevertheless, very few methods have been employed to solve the fault diagnosis problem till date. They include, Expert Systems based Computational intelligence techniques (Scientific Computation) such as, artificial neural networks (ANNs) [2] and genetic algorithms (GA) [3]. As the objective function is usually a second-order polynomial, Genetic Algorithm method has been employed to deal with such a problem [3]. Evolutionary Programming excludes crossover operations and hence have a shorter run time when compared to GA [3]. Faulted-section determination has been determined using model based reasoning in [4]. This calls for larger investments into protection models and knowledge engineering. Further scientific review defines the solution for fault location using Artificial Neural Nets. Many research groups have applied ANN [2], by using data from any one power line terminal, thus reducing the amount of required information. Reference [5] uses Bayes Theorem and applies a probabilistic model to the solution of a complex communication system.

A continuous escalation in the complexity, size, and reliability of modern industrial systems necessitates an advanced development of the control and fault diagnosis theory and practice. These requirements extend beyond normally accepted critical systems of the existing power stations/grid. As it is obvious, the controlled system is the main part of the scheme, and it is composed of actuators, process dynamics and sensors. Each of these parts is affected by several unknown inputs/attenuation that can be perceived as process or measurement noise as well as external disturbances acting on the system. When model-based control and diagnosis is utilized, then the unknown input can also be extended by model uncertainty via Gaussian/random operators, i.e., the mismatch between a model and the system being considered. The system could also be affected by faults, which can be divided into three primary groups, i.e., actuator faults, component (or process) faults, and sensor based faults, redefining the problem out of scope of this paper. The role of the fault diagnosis portion is to conditionally monitor the system behaviour and to provide all possible information regarding the abnormal functioning of its components. As a result, the overall task of fault diagnosis consists of three subtasks: fault detection, isolation and systemic updating. In the field of power system fault diagnosis both hybrid and conventional methods are being used. In our work, waveform matching technique is used to identify the fault type and fault location. Recent work on this method involves harmony search [14]. Advanced metrics involve the use of Fuzzy ART Maps [17], FIRANN [18], Unsynchronized and noncontact magnetic field measurements [19, 20]. 


\section{Problem Formulation}

This paper utilizes the concept from Waveform matching and Evolutionary algorithms, and creates an interactive approach to optimize the fault location. The software discrepancies further add strength to the problem formulation. The subsequent section discusses the methods adopted.

\subsection{Waveform Matching Technique}

In general, Digital Fault Recorders (DFRs) or Smart Metering Devices can locate the fault site in a SMART Grid. Nevertheless it is evident that such analysis is not always precise due to natural disturbances in signals on account of attenuation in practical circuitry. This deviation from ideal state can occur in the form of cross inductances of $\mathrm{OH}$ lines, Overhead capacitances (line to ground or line to line), corona effects in co-axial cables, radio interference in the form of frequency broadcasts, surge impedance loading and so on and so forth. This calls for optimizing the fault location using Evolutionary Algorithms. In this paper Fault Diagnosis of a high-voltage transmission line (HVTL) considers three major tasks, namely, fault-type identification, fault distance location and fault resistance estimation. The diagnosis problem is formulated as a single objective 3 dimensional optimization problem. The optimization decision variables involved in a basic fault diagnosis problem, are fault length from the reference bus, ground resistance between faulted line and earth, and fault nature. The expected variables pertaining to the original waveform, are obtained from the DFRs placed at the receiving/sending end substation in a real time scenario. Another waveform set is obtained from MATLAB simulations or the ISPEN tool in real time simulations. The optimized variables are automatically readjusted as per the presented algorithm, according to the condition that both these waveforms extracted simultaneously from DFR/ATP and MATLAB simulations coincide i.e. superimposition of both waveforms. Hence, the term Waveform Matching. The paper has been formulated using this relatively simple idea as shown in figure 1 below.

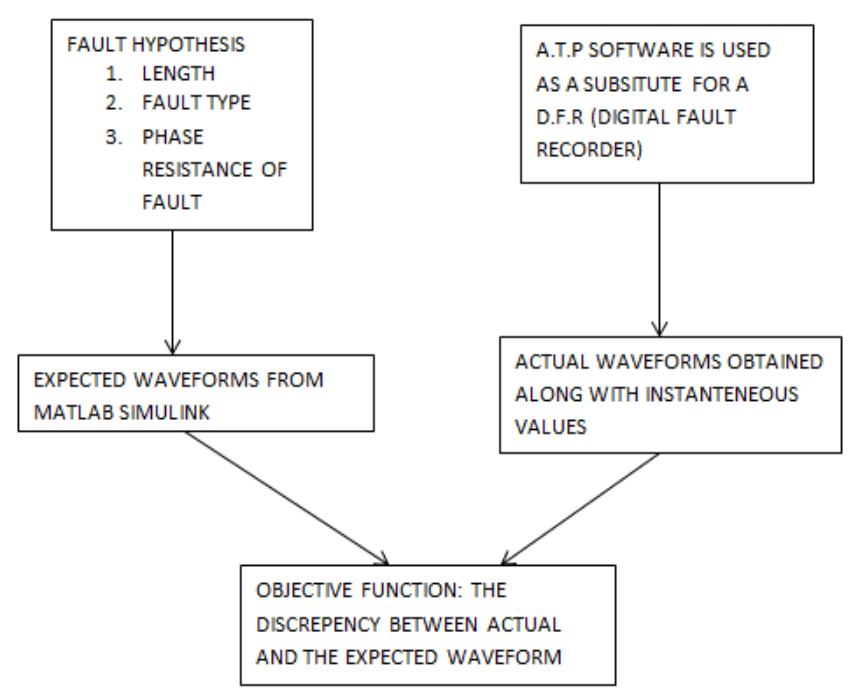

Figure 1. Paper Idea organization 


\subsection{Objective Function}

The waveform matching is done between the actual waveforms derived from the DFR and the expected waveforms produced from parallel simulation studies in MATLAB. A total of ' $M$ ' points in the actual and the expected waveforms in the range $\left[\mathrm{t}_{\mathrm{s}}, \mathrm{t}_{\mathrm{br}}\right]$ are sampled, respectively. An objective function (Error Function) that reflects the discrepancy between the actual and the expected waveforms is given as follows.

Error function $=\boldsymbol{f}(\boldsymbol{X})=\boldsymbol{f}\left(\boldsymbol{p}, \boldsymbol{d}, \boldsymbol{r}_{p}\right)=\sum_{i=A, B, C} \sum_{j=\mathbf{1}}^{M}\left|I_{i, j}-I_{i, j}^{*}(\boldsymbol{X})\right|$

where $\mathrm{i}=\mathrm{A}, \mathrm{B}$ or $\mathrm{C}$ represents phase $\mathrm{A}, \mathrm{B}$ or $\mathrm{C}$, respectively;

$\mathrm{j}$ is the $\mathrm{j}^{\text {th }}$ sampling point $(\mathrm{j}[\{1,2, \ldots, \mathrm{M}\}) ; \mathrm{Vi}, \mathrm{j}$ and Ii, $\mathrm{j}$ are the sample points of the duringfault voltages and currents in three phases, respectively, which are obtained from DFR in an actual fault scenario; $V_{j(X)} I_{j(X)}$, are the sample points of the during-fault voltage and current waveforms. These are produced by simulation studies corresponding to a given fault hypothesis $\mathrm{X}$ where $\mathrm{X}$ is the decision matrix to be optimized. Under Practical considerations, waveform matching is possible through actual waveforms derived from an alternate measuring device. An important point in fault diagnosis is taking into account the purpose and types of fault recording devices (characteristic features). The objective function in (1) is designated as a minimization function. The minimum value of $\mathrm{f}(\mathrm{x})$ is analogous to matched waveforms, hence the term waveform matching.

\section{Alternate Transient Program (ATP)}

Waveform matching is carried out by concurrently adjusting the waveforms from the recording devices and the simulation. But, in our analysis, it is impossible to adopt a DFR due to cost constraints and inflexibility in tuning the device. Instead of a DFR, ATP (Alternate Transient Program) has been employed as a substitute. ATP is used purposefully create a fault in a bus feeder and the waveforms of fault current and voltage are obtained with respect to time. These are the actual waveforms that represent the DFR recordings replaced with a software approach. Since ATP program is studies transient phenomenon through state space modelling, it is expected to mimic the station recorders. ATP contains a feature called COMTRADE which is basically used to transfer data sets between two Softwares. Expected waveforms are obtained from MATLAB and waveform matching between the waveform sets is done via. COMTRADE file format imported from ATP.

\subsection{COMTRADE Format}

COMTRADE (Common Format for Transient Data Exchange) is a file format created especially for transient simulations. It comprises library files and data files that store instantaneous values pertaining to a waveform. It is read and generated using GTPPLOT program. GTPPLOT is a plotting program for processing .PL4 output files of ATP-DRAW simulation results and converting their formats. It can be considered as a SMART platform to capture all data points from voltage and current probes placed in the circuit. It is compiled in GNU FORTRAN language, and makes use of the graphical package DISLIN. The ATP simulated data can be exported as PL4, COMTRADE, Matlab, Math Cad and Mathematical files. Furthermore, the program is used in calculations involving numerous Power Quality indices. Examples include Fourier series representation, estimation of Generator turbine shaft loss or life etc. The captured view of this program is as under. 
International Journal on Soft Computing (IJSC) Vol.4, No.4, November 2013

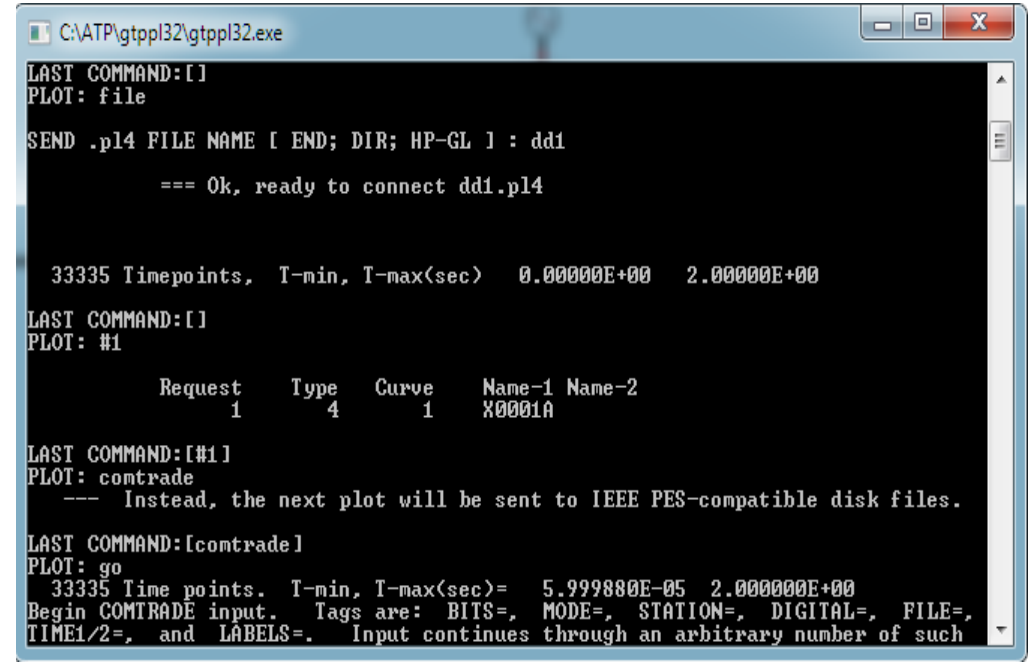

Figure 2. GTPPLOT Window

\section{EA BASED OPTIMIZATION TECHNIQUES}

In artificial intelligence, an evolutionary algorithm (EA) is a genre of evolutionary computation, a generic population-oriented meta-heuristic optimization algorithm. An EA uses mechanisms inspired by biological evolution/processes, such as reproduction, mutation, recombination, and selection. Candidate solutions of the optimization problem play the role of individuals in an interacting population, and the fitness function determines the environment/topography within which the solutions "live". Evolution of the population then takes place after the repeated application of the operators defined by mathematical interpretations. Evolutionary algorithms often perform well, thereby approximating solutions of all types of problems based on the underlying fitness landscape; this generality is shown by success in multitudes of fields such as robotics, operations research, management, power systems, supply chain management, political sciences, prediction, etc.

A large number of methods in meta-heuristics are constructed based on analogy of natural phenomena, such as biological evolution (Genetic Algorithm: GA), bird flocking or fish schooling (Particle Swarm Optimization: PSO) and behaviour of ants seeking a path (Ant Colony Optimization: ACO). A vast majority of heuristic and meta-heuristic algorithms have been derived from the behaviour of biological and/or physical systems in nature. For example, particle swarm optimization was developed based on the flocking swarm behaviour of birds and fish, while simulated annealing was based on the slow annealing process in metal fabrication. New algorithms have also emerged recently, like the harmony search, firefly algorithm et al. The former is inspired by the improvising process of composing a piece of music from strings, while the latter was formulated based on the flashing behaviour of fireflies of South American rainforest. Each of these algorithms have their own trade-offs. For example, simulating annealing will guarantee to find the exact optimal solution if the cooling process is slow enough and the simulation times are delayed.; however, the fine adjustment in certain constant parameters does affect the convergence rate of the optimization process. A feature of meta-heuristics is to have wide application range because information used in a search is only evaluation values. These methods are expected as convenient and powerful framework for practical problems from background of improvement of computer power nowadays. 


\subsection{Bare Bones Particle Swarm}

The Bare Bones swarm is conceptually the simplest of all variants of the PSO. Particle dynamics are replaced with sampling in a predefined probability distribution (normal distribution). The centre and dispersion of such a randomized allocation are set by the informers (local best particles) rather than global best. The Bare Bones Particle Swarm Optimizer is selected out for implementation in this paper because of its efficiency in search space exploitation. The conventional PSO adaptively follows the global best and local best solutions in the trajectory equation to define the solution space. Since it was later found that $\mathrm{G}_{\text {best }}$ particles could lead to premature convergence; here the local best particle dynamics is considered equally useful. The algorithm is stated below,

- For each variable $\mathrm{i}=1 \ldots \ldots \mathrm{N}$ do

○ $\overrightarrow{\mu_{\mathrm{I}}}=\mathrm{G}\left(\mathrm{N}_{\mu \mathrm{j}}\right) \quad ; \overrightarrow{\mu_{\mathrm{I}}}$ is the mean of the decision variable set $\mathrm{N}_{\mu \mathrm{j}}$

○ $\quad \vec{\sigma}_{1}=S\left(\mathrm{~N}_{\mu \mathrm{j}}\right) \quad ; \vec{\sigma}_{1}$ is the standard deviation of the associated set $\mathrm{N}_{\mu \mathrm{j}}$

- For each dimension $\mathrm{d}=1 \ldots \mathrm{D}$ do (Here $\mathrm{D}=3)$

$\circ \mathrm{x}_{\mathrm{d}}=\mathrm{F}\left(\mu_{\mathrm{jd}}, \sigma_{\mathrm{jd}}, \ldots.\right)$; normally distribute the new informers based on obtained dimensional mean and standard deviation

- End for

- $\overrightarrow{\mathrm{p}_{2}}+\mathrm{BEST}\left(\overrightarrow{\mathrm{x}}, \overrightarrow{\mathrm{p}_{2}}\right)$; derive local best particle for that particular iteration and then repeat

\subsection{BAT Algorithm}

This is a meta-heuristic algorithm relying on echolocation by bats. The algorithm is designed based on frequency of pulse emission, loudness and rate. In simulations, we use virtual bats naturally. In order to define the rules how their positions ' $_{\mathrm{i}}$ ' and velocities ' $\mathrm{v}_{\mathrm{i}}$ ' in a d-dimensional search space is updated, the following equations are used in a given time step, ' $t$ '.

$$
\begin{gathered}
\text { freq }_{i}=\text { freq }_{\min }+\beta \times\left(\text { freq }_{\max }-\text { freq }_{\min }\right) \\
\operatorname{vel}_{i}^{t}=v e l_{i}^{t-1}+\left(x_{i}^{t}-x_{\ddagger}\right) \times f_{i} \\
x_{i}^{t}=x_{i}^{t-1}+v e l_{i}^{t}
\end{gathered}
$$

Where $\beta \in[0,1]$ is a random number drawn from a uniform distribution. Here $x_{*}$ is the current global best location (solution: $\mathrm{g}_{\text {best }}$ ) which is identified after comparing all the solutions through successive substitutions among all the ' $n$ ' possible bats in the system. As the product of this algorithm is a velocity increment, we it to adjust the velocity change while fixing the other factor, depending on the type of the problem of interest. In this study, we will use $f_{\min }=0$ and $f_{\max }=100$, i.e. the rate of emission of ultrasonic pulses, depending on the problem scope. Initially at the start of the iterative procedure, each bat is randomly assigned a frequency which is drawn uniformly from a random distribution of $\left[f_{\min }, f_{\max }\right]$.

For the local search part, once a solution is selected from among the current best solutions, a new solution for each bat is generated locally using random walk simulations goven by $\mathrm{x}_{\text {new }}=\mathrm{x}_{\text {old }}+$ $A_{t}$, where $\beta \in[-1,1]$ is a random number, while $\mathrm{A}_{\mathrm{t}}=\left\langle\mathrm{A}_{\mathrm{t}}\right.$ and $\mathrm{I}>1$, is the average loudness of all the bats at this time step ' $t$ '. The velocity and positional update of bats have some similarity to the procedure in the standard particle swarm optimization as it essentially controls the pace and range of the movement of the particles in a swarm. 
As the loudness of pulse emission usually decreases once a bat has found its prey, while the rate of pulse emission increases, the loudness can be chosen as any value as per convenience. This ensures the adaptive feature of PSO where the population rate of convergence slows down upon detection of possible prey. For example, we can use $A_{0}=100$ and $A_{\min }=1$. For simplicity, we can also use $\mathrm{A}_{0}=1$ and $\mathrm{A}_{\min }=0$, assuming $\mathrm{A}_{\min }=0$ means that a bat has just found the prey and has temporarily rendered $\mathrm{UF}$ sound $=0$ decibels. Now we have the expression,

$$
A_{i}^{t+1}=\alpha A_{i}^{t}, r_{i}^{t+1}=r_{i}^{o}[1-\exp (-\gamma t)]
$$

where, $\alpha$ and $\gamma$ are constants. In fact, ' $\alpha$ ' is similar to the cooling factor of a condensing schedule in a simulated annealing process. For any $0<\alpha<1$ and $\gamma>0$, we have

$$
A_{i}^{t} \rightarrow 0, r_{i}^{t} \rightarrow r_{i}^{0} \text {, as } t \rightarrow \infty
$$

We can use $\alpha=\gamma$, and we have used $\alpha=\gamma=0.9$ in our simulations.

\section{Methodology}

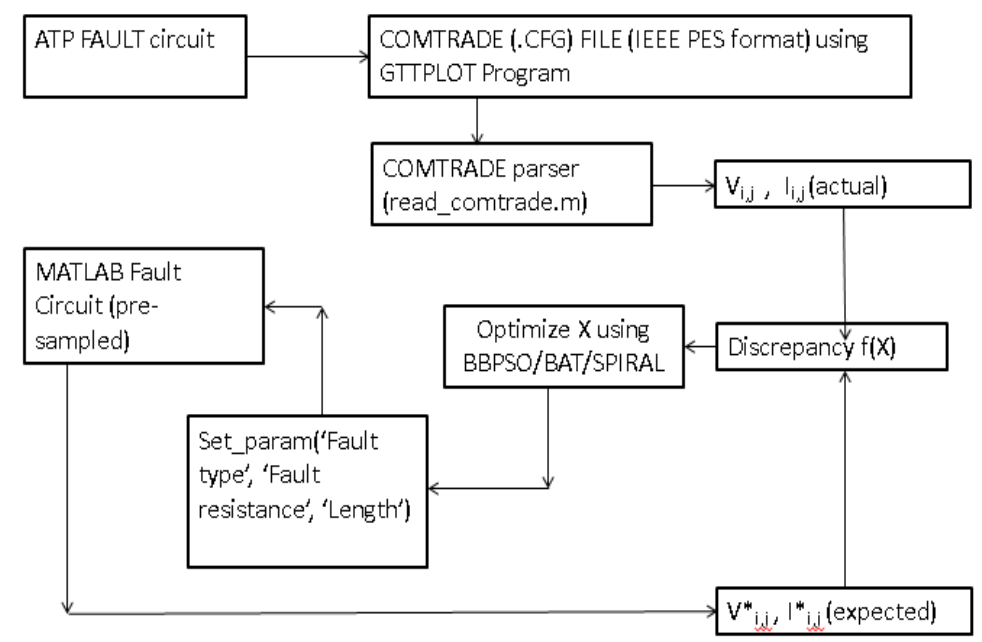

Figure 3. Schematic view of the proposed method

Actual (real time) waveforms are obtained from ATP-EMTP (Electromagnetic Transient Program) software and simulated waveforms are obtained using MATLAB (R2008b version). Parameters such as Voltage for PV buses, Load for PQ buses, transmission line parameters etc. are same for both simulations. Simulation in ATP is done using ATP-DRAW software developed by Bourneville Corporation. The circuitry is shown below for an IEEE 2 bus system between two generators $(\mathrm{U})$. 


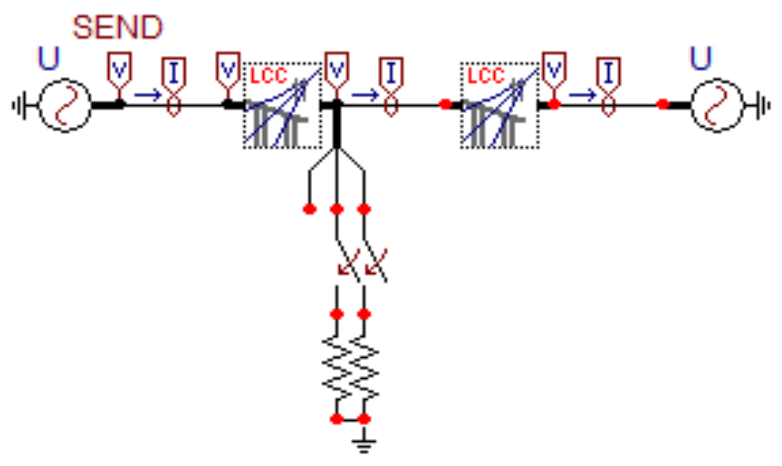

Figure 4. A 2 bus Fault Circuit Diagram in ATP

The ATP circuit is then decisively simulated with an integration time of 0.00002 seconds and maximum simulation time of 2 seconds. The fault triggering switches are then triggered under dynamic conditions i.e. when the circuit is under operational conditions and steady state values have seeped in. The switching interval timing is decided between 20 to 30 milliseconds i.e. the switch ON time is $20 \mathrm{msec}$ and the switch OFF time is $30 \mathrm{~ms}$. The current/default sampling rate in ATP creates around 1668 data points (instantaneous values) between this time interval. This represents the discretized version of the fault waveforms. The six waveforms needed for our analysis are $V_{a}, V_{b}, V_{c}$, (actual Voltages) $I_{a}, I_{b}$ and $I_{c}$ (actual Currents). COMTRADE data format for these waveforms (Common Method of Transient Data Exchange) is obtained from GTTPLOT, containing the individual data points in a structured format, along with other relevant details. Now, a similar circuit is created in MATLAB Simulink R2008b version as shown in the figure below, with similar parameters as its ATP counterpart.

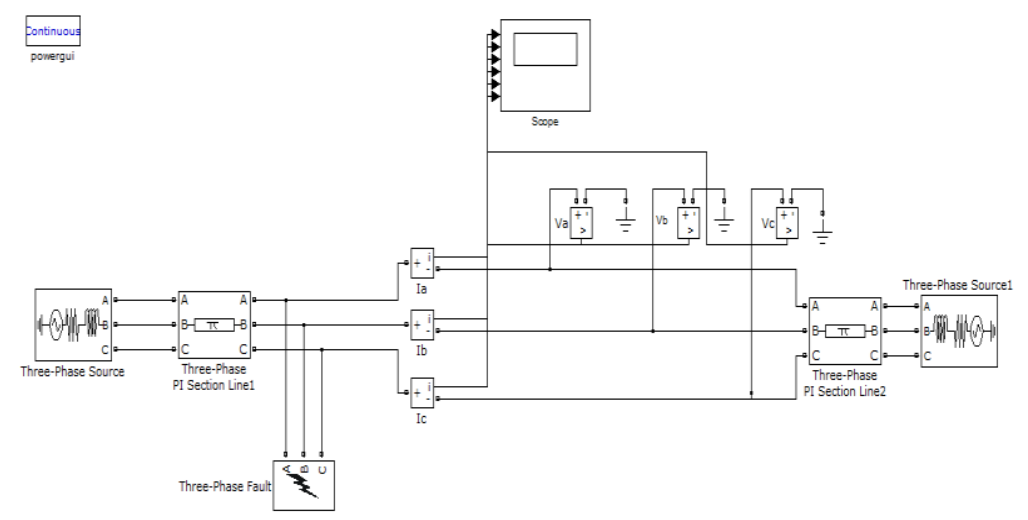

Figure 5. An equivalent 2 bus Fault Circuit Diagram in MATLAB

The above circuit is then simulated with few modified configuration parameters.

The following modifications are made in Simulink structure;

- output times in the range of [0:.00006:.35]

- ode45 (Dormand-Prince) solver

This simulation is called parallel from the main program via. the inbuilt function, 'set_param' function. 


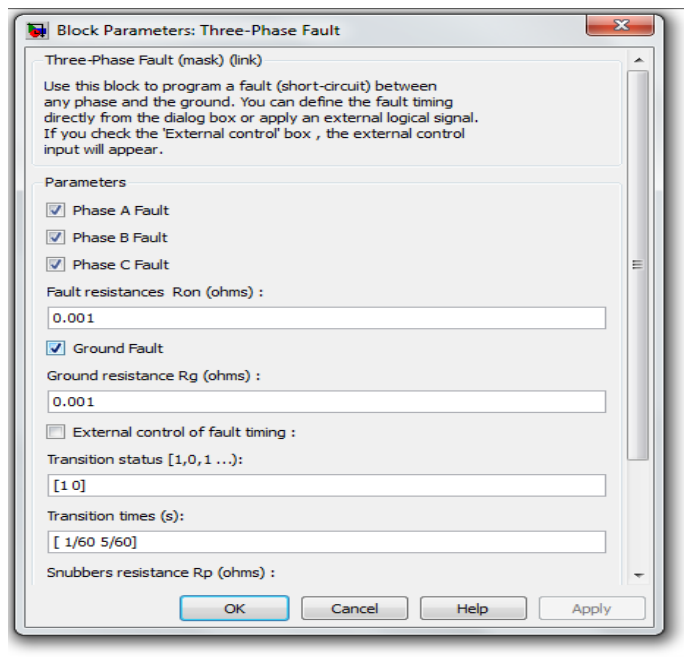

Figure 6. Three Phase Fault Block View

The ground resistance set to a default constant value of $0.008 \mathrm{ohm}$, and the type parameters are indicated by the fault type classification given in Table 5.1.

For 60 such cases simulated waveforms are obtained as shown in the Scope block.

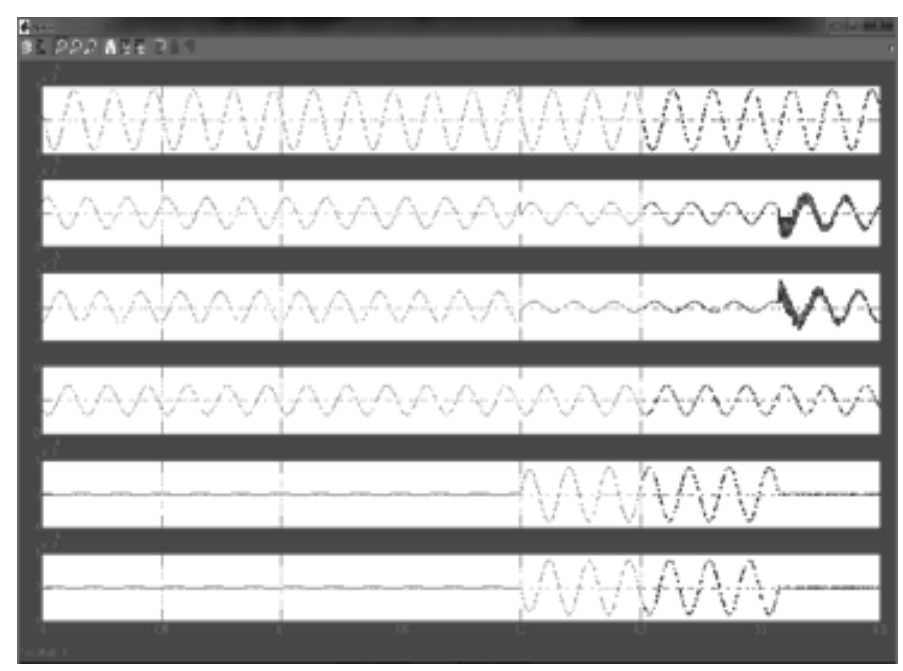

Figure 7. Fault Current and Voltage Waveforms

Now the optimization algorithm is run in the form of an iterative process for 40 iterations. Here the iteration count does not matter as the main aim of this analysis is to validate the effectiveness of a software as a substitute. Initially we devote around 12-15 iterations to precisely indicate the fault type and then the remaining 25-30 iterations to solve for the fault location and fault resistance, with the obtained fault type. This allows for reduced memory requirements and also sectionalizing the algorithm in two stages. The 3 dimensional co-ordinates then follow the search motion meeting the Optimization rules (explained earlier) according to this best fitness function. This updated matrix after the first iteration is now used to solve for the next iteration and so on. The procedure follows suite and is based on successive substitutions. The algorithm performance is escalated after dissecting the process into two sub-processes. This is because, after 15 iterations, all the elements of the third column meet at a point, i.e. the fault type. This is then used to determine the nature of the fault occurring in the system. It can also be used in special cases 
International Journal on Soft Computing (IJSC) Vol.4, No.4, November 2013

where only the fault type is desired to be known and hence reliability analysis needs to be done further on. The same algorithm is now run for a 2 dimensional problem as discussed above.

\section{RESULTS AND DISCUSSION}

It has been found that despite random initializations in the algorithm, the BBPSO gives near to an exact location of the fault. Slight distortions are found in raw fault signals from ATP software due to which, accurate match/superimposition is a problem for some of the waveforms. These problems can be curtailed using Wavelet based de-noising algorithms applied to ATP waveforms.

\subsection{Circuit Diagrams and Tabulation}

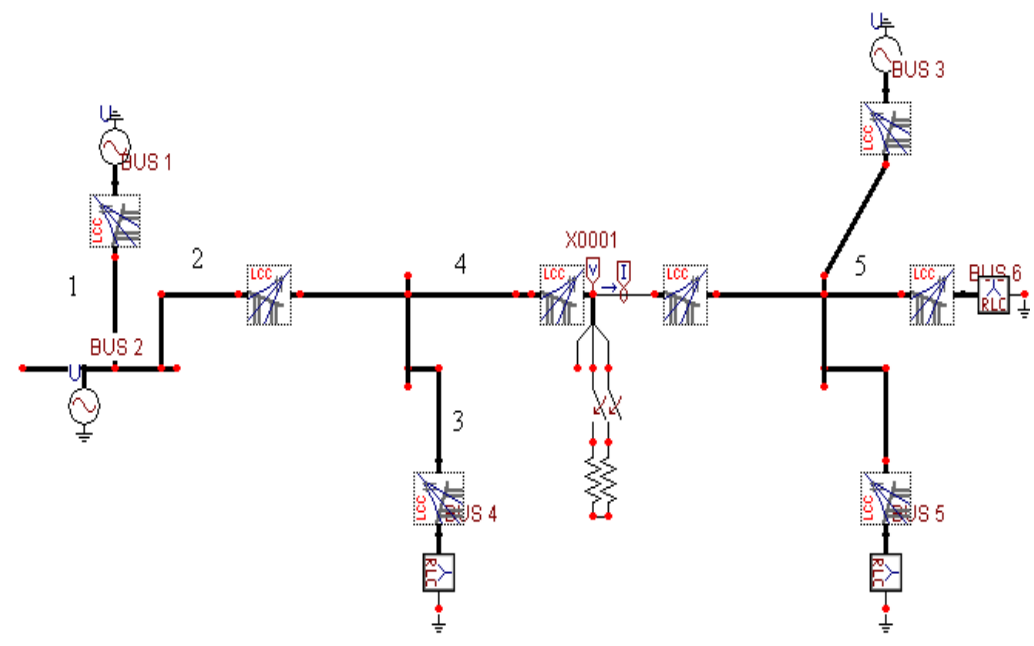

Figure 8. A 6 bus Fault Circuit in ATP

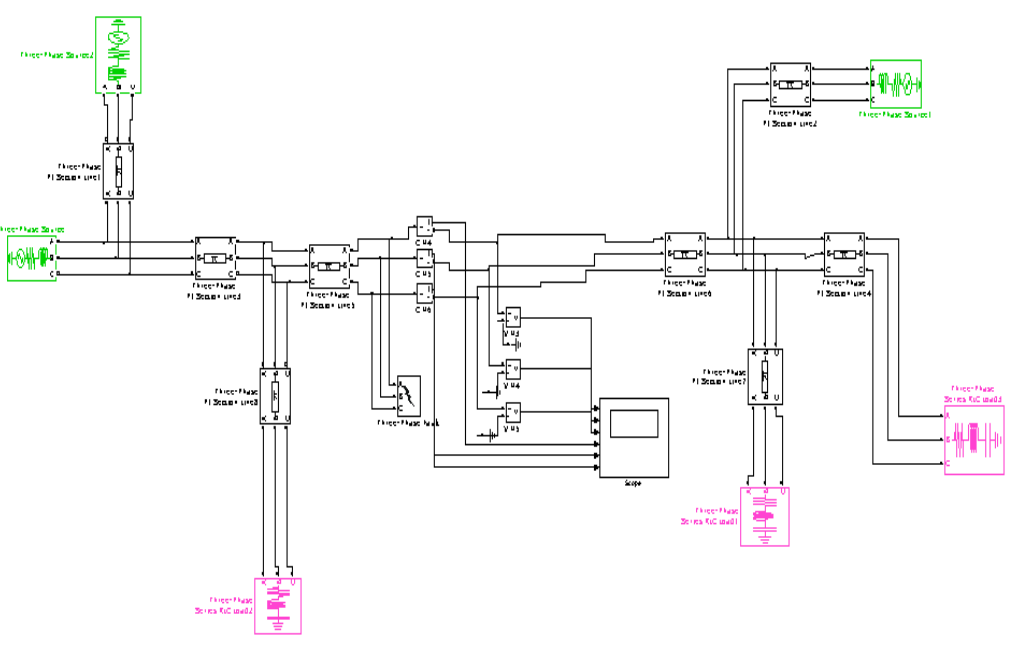

Figure 9. A 6 bus Fault Circuit in Matlab

The above circuit diagrams represent equivalent 6 bus systems in ATP and MATLAB software respectively. A line to line fault has been initiated in both the circuits. Each LCC block represents a transmission line setting in ATP-DRAW. 
International Journal on Soft Computing (IJSC) Vol.4, No.4, November 2013

Table 1. Parameter setting for 2 bus circuit

\begin{tabular}{|l|l|l|}
\hline Parameters & $\begin{array}{l}\text { Sending } \\
\text { End }\end{array}$ & Receiving End \\
\hline Set Voltage & $504 \mathrm{kV}$ & $502 \mathrm{kV}$ \\
\hline Set Source Resistance & $(0.5-1) \Omega$ & $(0.5-1) \Omega$ \\
\hline Set Source Inductance & $0.1-100 \mathrm{mH}$ & $0.1-100 \mathrm{mH}$ \\
\hline Set Positive Sequence Impedance & $0.108+\mathrm{j} 0.293$ & $0.108+\mathrm{j} 0.293$ \\
\hline Set Zero Sequence Impedance & $0.998+\mathrm{j} 1.295$ & $0.998+\mathrm{j} 1.295$ \\
\hline Set Positive Sequence Capacitance & $0.0121 \mu \mathrm{F} / \mathrm{km}$ & $0.0129 \mu \mathrm{F} / \mathrm{km}$ \\
\hline Set Zero Sequence Capacitance & $0.005 \mu \mathrm{F} / \mathrm{km}$ & $0.005 \mu \mathrm{F} / \mathrm{km}$ \\
\hline
\end{tabular}

Table 2. Parameter setting for a 6 bus system

\begin{tabular}{|l|l|}
\hline Parameters & Generator Bus \\
\hline Set Voltage & $110 \mathrm{kV}$ \\
\hline Set Source Inductance & $0.1 \mathrm{mH}$ to $150 \mathrm{mH}$ \\
\hline Set Source Resistance & $0.5 \mathrm{~m} \Omega$ \\
\hline Set Phase Angle & -20 to 20 \\
\hline Parameters & Load Bus \\
\hline Set Real Power & $20 \mathrm{Kw}$ \\
\hline Set Reactive Power & $100 \mathrm{VAR}$ \\
\hline
\end{tabular}

Table 3. Variable Declaration

\begin{tabular}{|l|l|}
\hline Fault Type & Variable \\
\hline Single Line to Ground (A-Ground) & 1 \\
\hline Single Line to Ground (B-Ground) & 2 \\
\hline Single Line to Ground (C-Ground) & 3 \\
\hline Line to Line (A-B) & 4 \\
\hline Line to Line (B-C) & 5 \\
\hline Line to Line (A-C) & 6 \\
\hline Double Line to Ground (A-B-Ground) & 7 \\
\hline Double Line to Ground (B-C-Ground) & 8 \\
\hline Double Line to Ground (A-C-Ground) & 9 \\
\hline Three phase Line to Line (A-B-C) & 10 \\
\hline Three phase to Ground (A-B-C-Ground) & 11 \\
\hline
\end{tabular}

Table 4. Results for 2 bus system using BBPSO and BAT algorithm

\begin{tabular}{|c|c|c|c|c|c|}
\hline \multicolumn{6}{|c|}{ Using BBPSO } \\
\hline \multicolumn{3}{|c|}{ Actual Values } & \multicolumn{3}{|c|}{ Diagnostic Values } \\
\hline Fault Type & Length & Fault Resistance & Fault Type & Length & Fault Resistance \\
\hline 5 & 80 & 1.5 & 5 & 80 & 1.4245 \\
\hline 7 & 30 & 1.4 & 7 & 29.2 & 1.37 \\
\hline \multicolumn{6}{|c|}{ Using BAT-Algorithm } \\
\hline 6 & 80 & 1.3 & 6 & 80.65 & 1.45 \\
\hline 2 & 40 & 1.5 & 2 & 39.96 & 1.0 \\
\hline
\end{tabular}




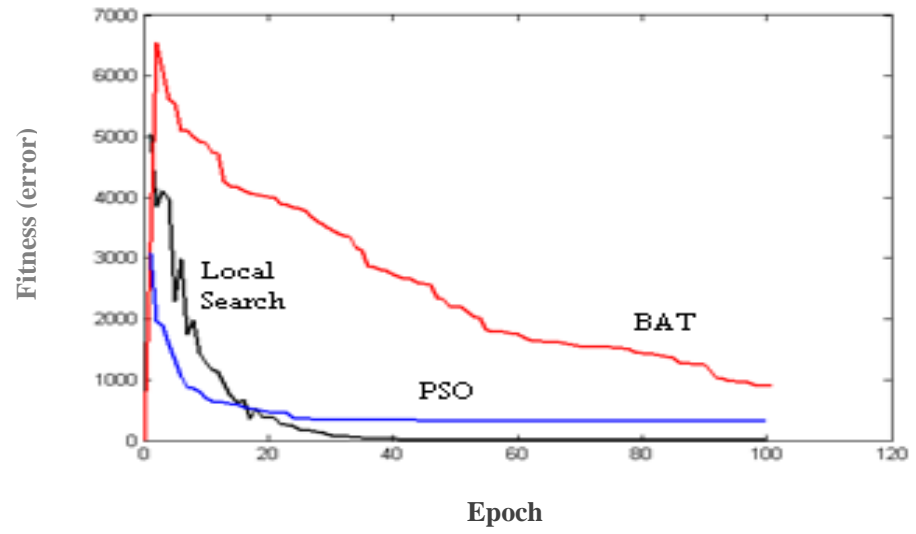

Figure 11. Fitness vs. iteration count

The convergence characteristics shown below indicate that Local Search (BBPSO) is a better algorithm in terms of precision. Moreover, the presence of random rate of emissions, after every iterations poses a problem as every time a random initialization produces a different fault location. Such is not the case with Bare Bones, where the line constant to ' $x$ ' axis indicates an exact location. Computation times are severely affected in BAT optimization, hence, further analysis has been focussed on BBPSO, in less time.

Table 5. Results for BBPSO

\begin{tabular}{|c|c|c|c|c|c|c|}
\hline \multirow[b]{2}{*}{$\begin{array}{l}\text { Transmission } \\
\text { line }\end{array}$} & \multicolumn{3}{|c|}{ Actual Values } & \multicolumn{3}{|c|}{ Diagnosis Values } \\
\hline & Fault Type & Length & $\begin{array}{l}\text { Fault } \\
\text { Resistance }\end{array}$ & Fault Type & Length & $\begin{array}{l}\text { Fault } \\
\text { Resistance }\end{array}$ \\
\hline \multicolumn{7}{|l|}{ For 6 bus } \\
\hline 1 & 7 & 40 & 1.5 & 7 & 39.53 & 1.4776 \\
\hline 2 & 11 & 70 & 1.7 & 11 & 70.45 & 1.3257 \\
\hline 3 & 9 & 10 & 1.2 & 9 & 9.65 & 1.621 \\
\hline 4 & 5 & 95 & 1.7 & 5 & 93.3 & 1.652 \\
\hline 5 & 11 & 15 & 1.9 & 11 & 15.73 & 1.9484 \\
\hline \multicolumn{7}{|l|}{ For 14 bus } \\
\hline 1 & 1 & 40 & 1.5 & 1 & 40.03 & 1.56 \\
\hline 2 & 4 & 60 & 1.2 & 4 & 59.34 & 1.25 \\
\hline
\end{tabular}

The diagram for 14 bus is provided in Appendix. BBPSO tends to converge the solutions into a better optimal condition with greater precision. Analysis showed that the length had converged soon enough compared to fault resistance. The length had converged to the actual length specified in ATP, i.e. $50 \mathrm{~km}$, in the $7^{\text {th }}$ iteration itself. It was found that initially the fault length from one of the buses assumed as reference had converged to $56.780 \mathrm{~km}$ after $3^{\text {rd }}$ iteration. But the resistance showed oscillations between 1.66 and 1.5. This conclusively states that fault resistance and length are mutually responsible for accurate location algorithms and the process is dynamic in nature. Waveforms have matched after gradual shifts as per iteration build up indicated in Figure 11. At the end of the final iteration all the parameters corresponding to this matched/coinciding waveform is the optimized fault setting that we are looking for. 
International Journal on Soft Computing (IJSC) Vol.4, No.4, November 2013

An unusual hindrance in our analysis was the occurrence of distortions and phase drifts due to software make up. This problem has been clearly articulated in the set of figures. Such phenomenon is seen due to different starting times for the two waveforms. Fluctuations in the form of Gaussian noise in ATP simulations and overall d.c. component shift (momentary fault currents) lead to inefficiency in analysis. This however is resolved via. trial and error setting of source inductances, phase angle and fault resistance in matlab circuit.

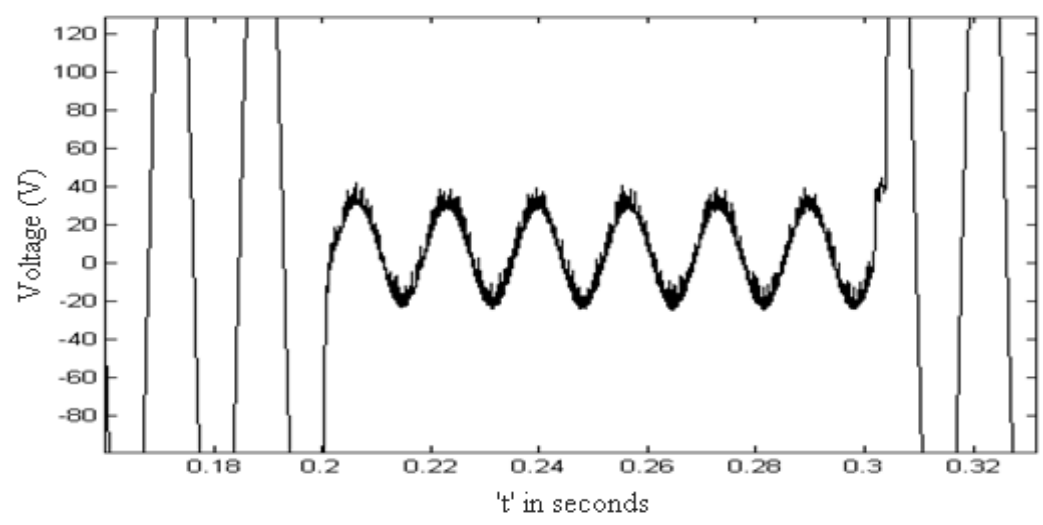

Figure 12(a). Distortion in fault waveform due to high Inductance

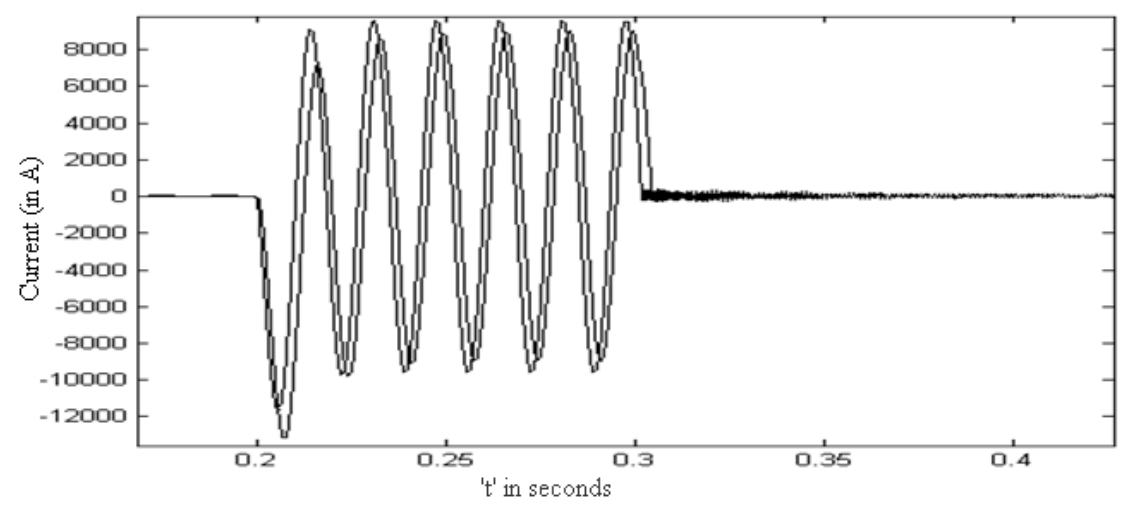

Figure 12(b). Phase angle shifting in fault waveform

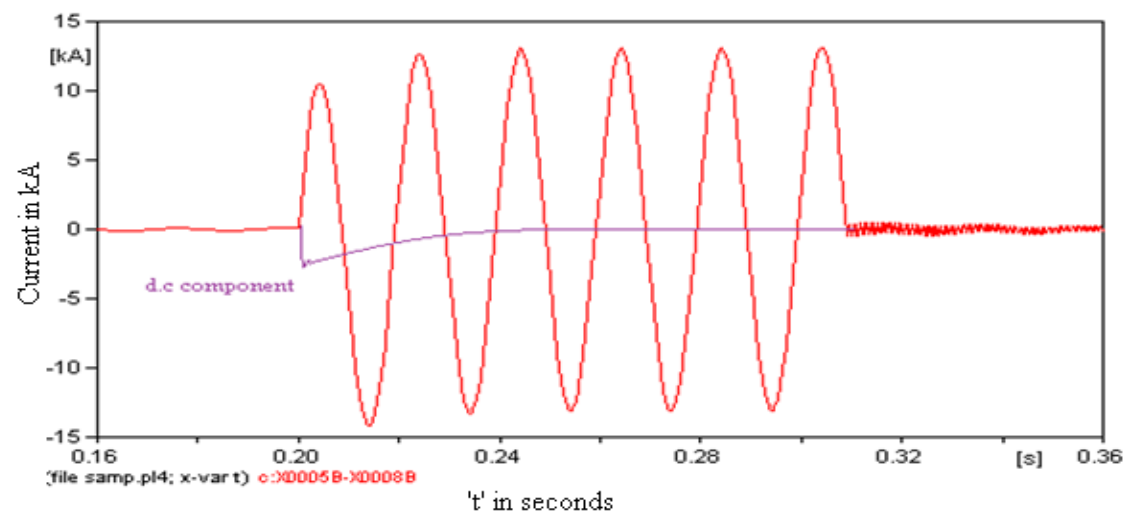

Figure 12(c). D.C Component mismatch due to momentary currents 
The range of values given in Table II and III, obtained via inspection is used in this analysis. DFRs are accurately configured and so this problem does not arise in Real time situations. Noise and drifts can be simply eliminated using circuit based filtering and pre-amplification. But accurate configuration setting is needed prior to installemnt in area. Even in this analysis, the problem in Figure 12(a) could be resolved through de-noising. But, fundamentally we are interested in waveform matching of un-manipulated raw signals. The problem of phase shifts occurs due to load variations and even point of origin of these waveforms. This can be assumed to be in phase, and changes in source phase angle needs to be made in matlab, indicating subtransient and transient reactance drops with internal emfs. The presence of momentary currents in Figure 12(c) cannot be circumvented, and so is the primary source of deviations in error function.
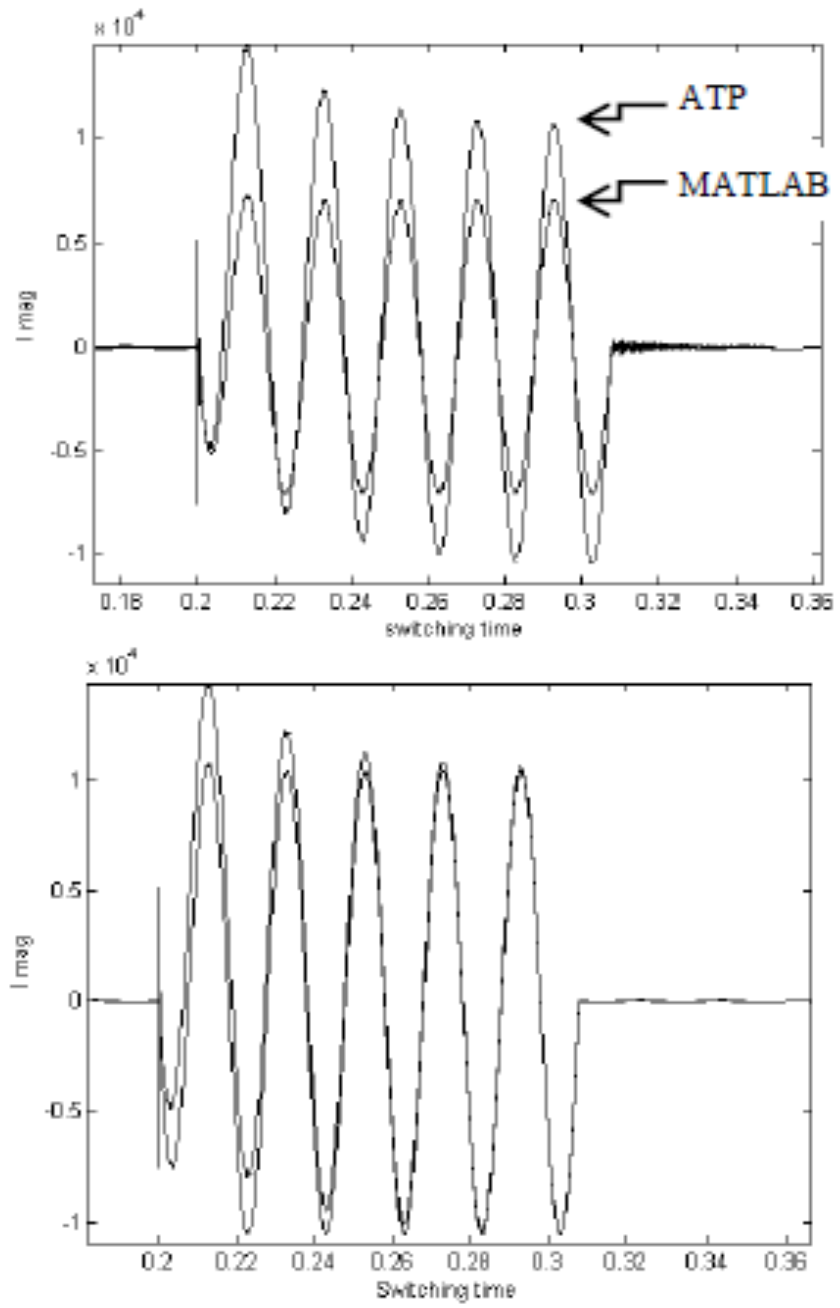

Figure 13. Accurate Waveform Matching

However, in most cases an accurate fault optimization would correspond to exact waveform superimposition as shown in the figure above. It is under such optimized parameter settings that the error function reduces to zero and hence, the term waveform matching. 


\subsection{Performance Evaluation}

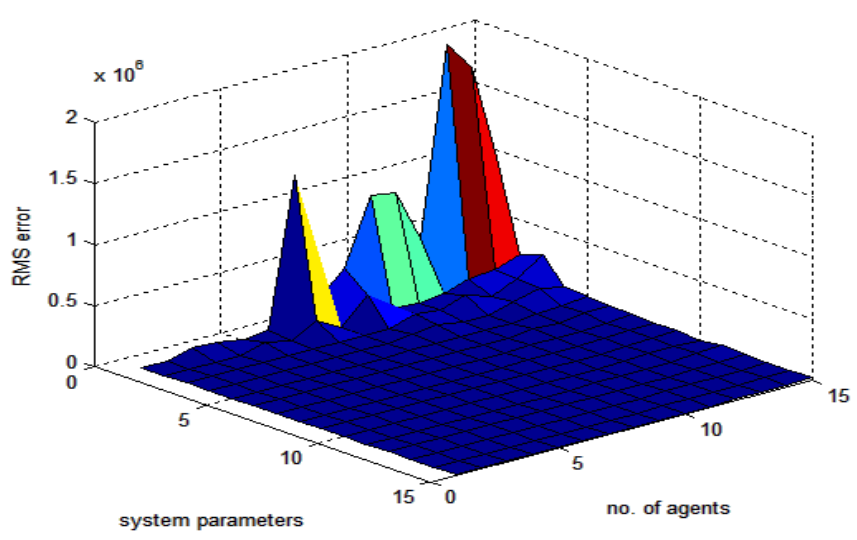

Figure 14. Performance of BBPSO with variation in Agents and Fault indicating parameters.

The above three dimensional representation clearly states the relationship between the Root Mean Square Error/Fitness function with number of system fault indicating parameters. It can be seen that parameters such as;

- Voltage readings

- Current readings

- CB switching times

- Relay impedance measured through Megger circuits

- Phase angles of the sequence components

and many more systemic parameters can be obtained from metering equipment along with the particle count used for running the computations. Bare Bones shows better performance under the presence of lesser such fault indicating parameters stated above and higher particle/agent count. This cuts down on expenses in procuring such measuring devices and assists in economic fault diagnosis. As observed, system performance deteriorates with lesser agents and higher parameters. This is obvious as more number of informers/local particles are needed to determine the best local solutions. A major complication to the application of this particular algorithm in real time scenarios is memory constraints, and work is currently underway with other algorithms to replace the same.

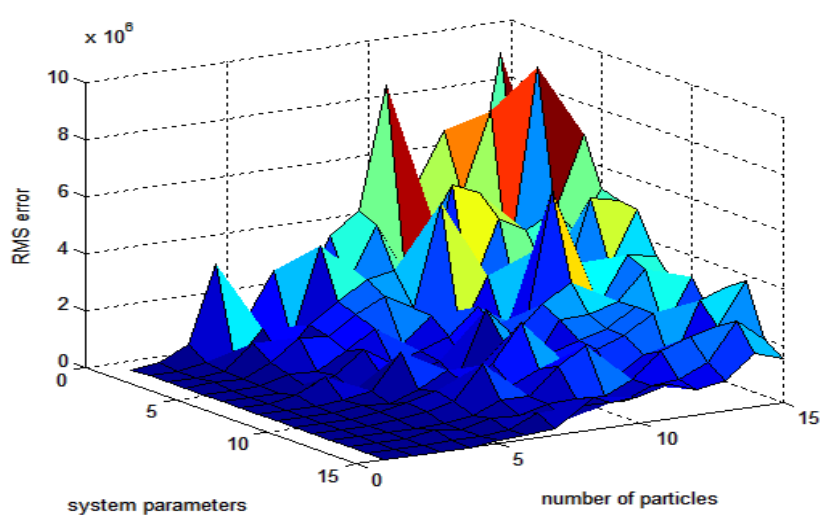

Figure 15. Performance of BAT with variation in Agents and Fault indicating parameters. 
BAT algorithm performs well for any agent count and lesser number of fault indicating parameters. This is obvious as the plot increases gradually from one end of the three D space and performance issues are egregious at the higher system parameter state and lower agent count. So, in terms of Power System Reliability studies, BBPSO is better compared to Bat algorithm.

\section{CONClusion}

Evolutionary Optimization Algorithms can be successfully implemented for accurate fault diagnosis in a double circuit HVTL. It has been proven that waveform matching approach is an effective tool for identifying fault parameters such as length, fault type and fault resistance. This is justified as captured waveforms from two variants superimpose over one another at the optimized parameter setting conditions, when the error function is minimum. The software proposed (Alternate Transient Program) has been used successfully to mimic the real time behaviour of Digital Fault Recorders (DFRs) in substations. The results were analysed for an IEEE 2 bus, 6 bus and 14 bus system using BBPSO algorithm. As far as Power System Reliability is concerned, Bare Bones Particle Swarm Optimization can be used, as local best solutions (informers) give a precise trajectory towards the minimal value of error, compared to global best solutions of BAT as is evident from the convergence characteristics. As observed, waveform distortions of Gaussian nature and phase angle drifts lead to misleading results in some of the transmission lines (especially lines between two adjacent load buses), conditional tuning of the source inductance and phase angle settings is found to be a pre-requisite for such systems to counter the effects of software mismatches. This technique will be able to facilitate in the creation a knowledge base to have the same technique of waveform matching using evolutionary algorithms run successfully on power grids using expert systems. Furthermore, as per fault resistance values, the accurate relay setting of impedance based relays can be tuned for fault isolation/clearance. The underlying future scope of the project will be incorporation of this approach in real life macro power grid systems using better metering equipments and supercomputers. Though time consuming, it will assist in the creation of an adaptive look up table as a reference medium for TSOs to act during crisis. Furthermore, work is underway with application of Neigh Block thresholding schemes and Stein's unbiased risk estimator for denoizing these waveforms and obtaining accurate matching. This will solve the problem of tuning sources as per requirement.

\section{APPENDIX}

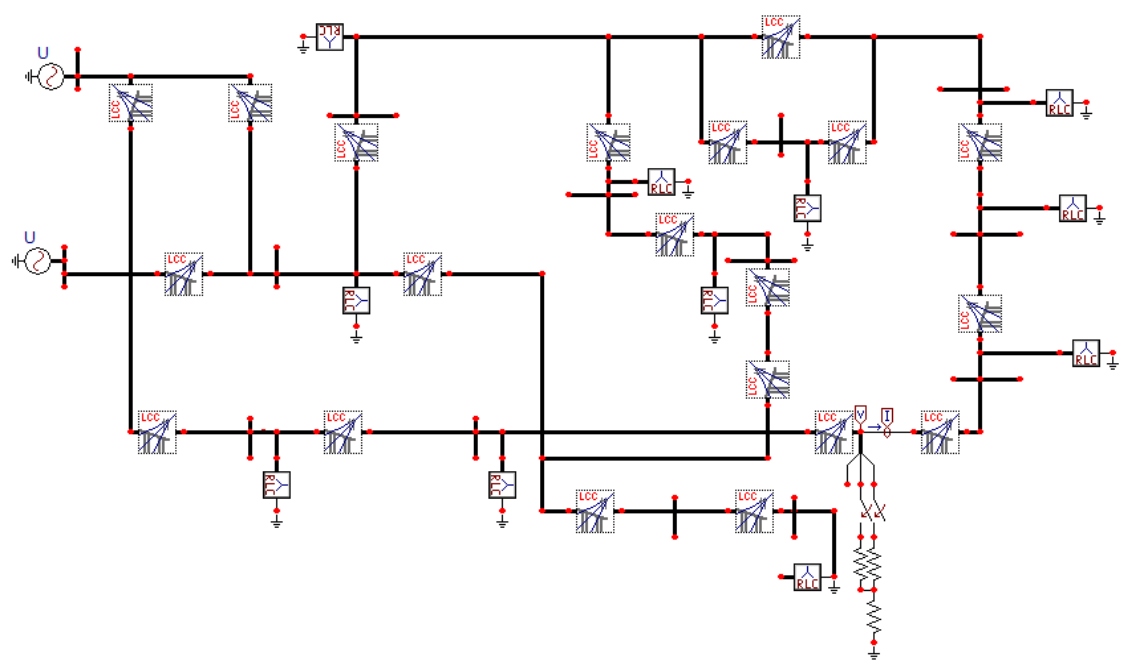




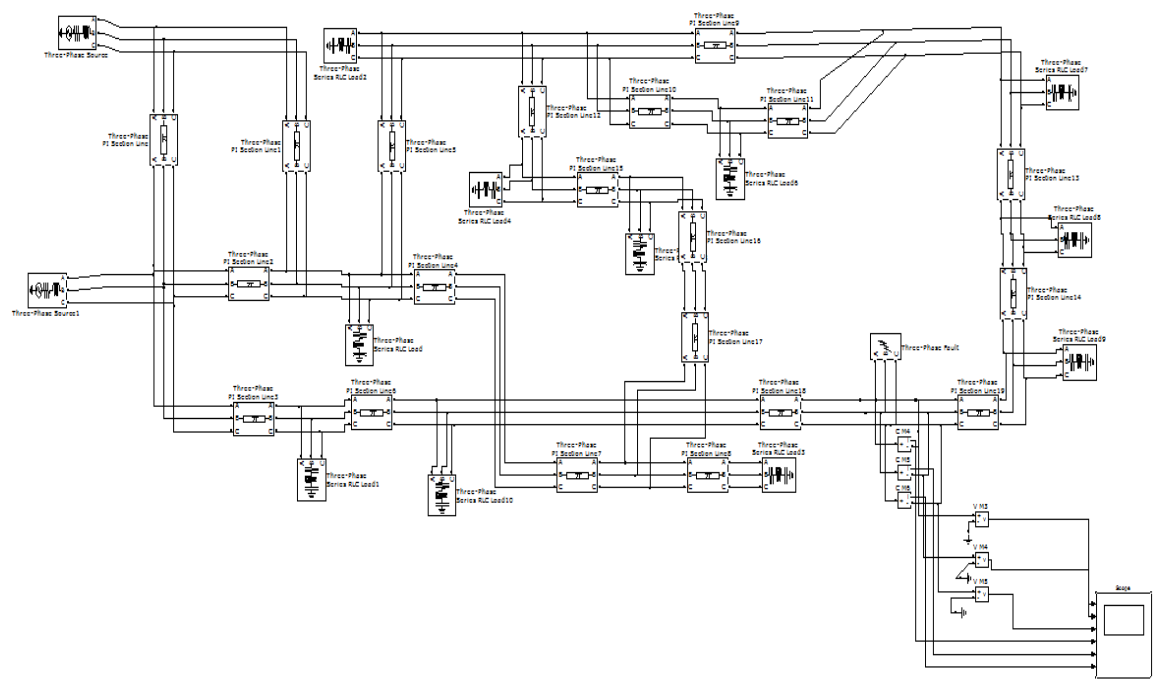

Figure 16. ATP and MATLAB 14 bus system

\section{ACKNOWLEDGEMENTS}

The authors would like to thank SRM University and other faculty members from EEE Dept. of SRM University for their support and encouragement. Special thanks to Head of Department, Dr. S.S.Dash and M.Tech faculty for giving necessary inputs in this project.

\section{REFERENCES}

[1] Ashish Ghosh, Sachinadanda, "Evolutionary Algorithm for multi criterion optimization: A survey", vol 2, No 1, April 2004 Indian Statistical Institute

[2] Steinder M., Sethi S. "Probabilistic Fault Localization in Communication Systems Using Belief Networks", IEEE/ACM Transactions on Networking, Vol. 12, No. 5, October 2004

[3] D. E. Goldberg, "Genetic Algorithms in Search, Optimization and Machine Learning", AddisonWesley, 1989.

[4] Ahemd El-Betar, MagdyM. Abdelhamed, Ahmed El-Assal and Roubi Abdelsatar "Fault Diagnosis of a Hydraulic Power System Using Artificial Neural Network” JKAU: Eng. Sci., Vol. 17 No. 1, pp: 117 - 137 (2006A.D. /1427 A.H.)

[5] P. D. Yehsakul, I. Dabbaghchi, "A topology-based algorithm for tracking network connectivity", IEEE Trans. on Power Delivery, vol. 10, no. 1, Feb. 1995, pp. 339-346.

[6] D. Novosel, D. Hart, E. Udren, and J. Garitty, "Unsynchronized one terminal fault location estimation”, IEEE Trans. on Power Delivery, vol. 11, no. 1, Jan. 1996, pp. 130-138.

[7] Christian M. Schegan, "A Hardware/Software Platform for Fault Detection and Identification in Electric Power Distribution Systems for Testing Various Detection Schemes" A Thesis Submitted to the Faculty of Drexel University, SEP 2008.

[8] M. Clerc, J. Kennedy, "The particle swarm: Explosion, stability and convergence in a multidimensional space," IEEE Trans. Evolutionary Computation, vol. 6, no. 1, pp. 58-73, Feb. 2002.

[9] R. Poli, "Mean a, herds and schools: A distributed behavioral model," Computational Graph., vol. 21, no. 4, pp. 25-34, 1987.

[10] R. Eberhart, Y. Shi, and J. Kennedy, Swarm Intelligence. San Mateo, CA: Morgan Kaufmann, 2001.

[11] J. Pẽna, "Theoretical and empirical study of particle swarms with additive stochasticity and different recombination operators," in Proc. 10th Annual Conference GECCO, 2008, pp. 95-102.

[12] J. Pe na, "Simple dynamic particle swarms without velocity," in Ant Colony Optimization and Swarm Intelligence (LNCS 5217). Berlin/Heidelberg, Germany: Springer, 2008, pp. 144-154.

[13] T. M. Blackwell and D. Bratton, "Examination of particle tails," J. Artificial Evolutionary Applications, vol. 2008, no. 893237, p. 10, 2008. 
International Journal on Soft Computing (IJSC) Vol.4, No.4, November 2013

[14] L.Wei, W.Guo, F.Wen, G.Ledwich, Z.Liao, J.Xin "Waveform matching approach for fault diagnosis of a high voltage transmission line employing harmony search algorithm". IET Generation Transmission Distribution. 2010, Vol. 4, Iss.7, pp. 801-809.

[15] Joe Perez, "A Guide to Digital Fault Recording Event Analysis", ERL Phase Power Technologies, Winnipeg, MB, R3Y 1G4, Canada

[16] IEEE Std C37.111-1999, "IEEE Standard Common Format for Transient Data Exchange (COMTRADE) for Power Systems", Approved 18 March 1999

[17] Aggarwal, R.K.; Xuan, Q.Y.; Johns, A.T. ; Furong Li, "A novel approach to fault diagnosis in multicircuit transmission lines using fuzzy ART map neural networks", Neural Networks, IEEE Transactions on (Volume:10, Issue: 5 ), pp $1214-1221$

[18] Fernandez, A.L.O. ; Ghonaim, N.K.I., "A novel approach using a FIRANN for fault detection and direction estimation for high-voltage transmission lines", Power Delivery, IEEE Transactions on (Volume:17, Issue: 4 ), pp $894-900$

[19] Korres, G.N.;, Apostolopoulos, C.A., "Precise fault location algorithm for double-circuit transmission lines using unsynchronised measurements from two anti-parallel ends", Generation, Transmission \& Distribution, IET (Volume:4, Issue: 7 ), July 2010, pp $824-835$

[20] Qi Huang ;, Wei Zhen ;, Pong, P.W.T., "A Novel Approach for Fault Location of Overhead Transmission Line With Noncontact Magnetic-Field Measurement", Power Delivery, IEEE Transactions on (Volume:27, Issue: 3 ), July 2012, pp 1186 - 1195

\section{Author}

Ripunjoy Phukan received his B.Tech degree in Electrical and Electronics Engineering from SRM University, Chennai in 2013.Presently, he is pursuing a research internship at Indian Institute of Technology, Guwahati working on PHEV integration into Power Grids. His research interest include Power Systems Optimization, Intelligent control of systems, Condition monitoring in insulation systems.

A Rathinam received his Master's degree from Annamalai University, Chennai, in 1997. Presently he is an assistant professor at SRM University, EEE department. His research interests include Power systems Optimization, Signals \& systems Multidimensional systems, Microprocessors, Intelligent Controllers, Soft computing \& evolutionary methods.
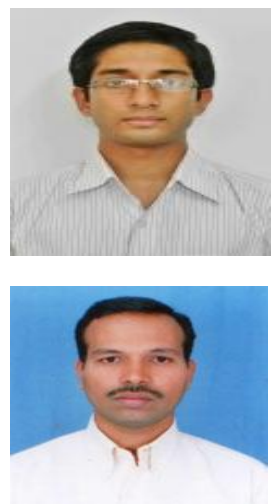

Rishab K Gupta received his B.Tech degree in Electrical and Electronics Engineering from SRM University, Chennai in 2013. He is currently pursuing graduate studies in Power Systems at University of Texas, Dallas.

Sandeep Dadga received his B.Tech degree in Electrical and Electronics Engineering from SRM University, Chennai in 2013. His research interests revolve around IT based solutions in Engineering. 